\title{
Gravitational fragmentation of the Carina Flare supershell
}

\author{
Richard Wünsch \\ Astronomical Institue of the ASCR, Boční II 1401, 14100 Prague, Czech Republic \\ email: richard@wunsch.cz
}

\begin{abstract}
We study the gravitational fragmentation of a thick shell comparing the analytical theory to 3D hydrodynamic simulations and to observations of the Carina Flare supershell. We use both grid-based (AMR) and particle-based (SPH) codes to follow the idealised model of the fragmenting shell and found an excellent agreement between the two codes. Growth rates of fragments at different wavelength are well described by the pressure assisted gravitational instability (PAGI) - a new theory of the thick shell fragmentation. Using the APEX telescope we observe a part of the surface of the Carina Flare supershell (GSH287+04-17) in the ${ }^{13} \mathrm{CO}(2-$ 1) line. We apply a new clump-finding algorithm DENDROFIND to identify 50 clumps. We determine the clump mass function and we construct the minimum spanning tree connecting clumps positions to estimate the typical distance among clumps. We conclude that the observed masses and distances correspond well to the prediction of PAGI.
\end{abstract}

Keywords. ISM: bubbles, ISM: molecules, stars: formation
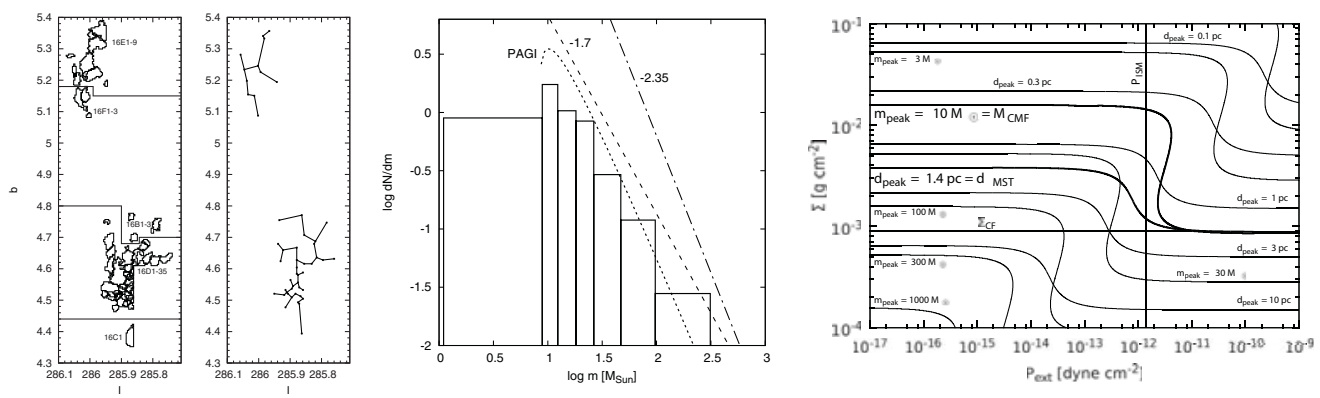

Figure 1. From left to right: (i) contours of clumps identified in the observed region; (ii) minimum spanning tree used to measure the mean distance among clumps; (iii) clumps mass spectrum compared with the prediction of the PAGI theory (Wünsch et al. 2010); and (iv) position of the CMF peak (solid contours) and the typical distance among clumps (dashed contours) as functions of the supershell surface density and the external pressure according to PAGI. Contours for the observed values intersect close to values expected for the Carina Flare supershell (dash-dotted lines). See Wünsch et al. (2012) for details.

\section{Acknowledgements}

This work is supported by the project P209/12/1795 of the Czech Science Foundation and by the project RVO:67985815.

\section{References}

Wünsch, R., Dale, J. E., Palouš, J., \& Whitworth, A. P. 2010, MNRAS 407, 1963

Wünsch, R., Jáchym, P., Sidorin, V., et al. 2010, A\&̈A 539, A116 